\title{
Vol. 67, No. 4
}

In the report "Outbreak of Seoul Virus Among Rats and Rat Owners - United States and Canada, 2017," on page 132 , the second sentence of the first paragraph under "Public Health Response," should have read "On February 10, 2017, the World Health Organization was notified of the U.S. and Canadian infections and investigations as required by International Health Regulations. ${ }^{\$ ”}$ 